\title{
La prótesis de pene en el tratamiento de la disfunción eréctil
}

\author{
G. Atienza Merino \\ Axencia de Avaliación de Tecnoloxías Sanitarias de Galicia (avalia-t) \\ Consellería de Sanidade. Edif. Administrativo de San Lázaro. Santiago de Compostela.
}

Actas Urol Esp 2006; 30 (2): 159-169

\section{RESUMEN}

LA PRÓTESIS DE PENE EN EL TRATAMIENTO DE LA DISFUNCIÓN ERÉCTIL

La disfunción eréctil es una patología que, con diferentes grados de intensidad, afecta a cerca del $20 \%$ de los varones adultos españoles. Su tratamiento se realiza habitualmente de forma escalonada, reservándose las prótesis de pene para cuando han fracasado otros tratamientos previos.

El objetivo de este trabajo es evaluar, de acuerdo al estado de conocimiento actual, la efectividad y seguridad de las prótesis de pene en el tratamiento de la disfunción eréctil. Para ello se seleccionaron 52 artículos, observándose porcentajes de supervivencia protésica a los 5 años del 78-91\% y siendo las complicaciones quirúrgicas del 3-8\%. Los fallos mecánicos y la infección fueron menores en las prótesis semirrígidas que en las hinchables, obteniéndose altos niveles de satisfacción postoperatoria, tanto entre los pacientes como en sus parejas, mayores incluso que con el resto de tratamientos disponibles en la actualidad.

La implantación de una prótesis de pene debe reservarse para la disfunción eréctil de carácter orgánico en la que hayan fracasado otros tratamientos previos, debiendo valorarse la relación riesgo/beneficio e informando al paciente de los resultados que se esperan conseguir y de las posibles complicaciones que puedan surgir.

La mayor preocupación de nuestra sociedad con respecto a la función eréctil, unido a la disponibilidad de fármacos eficaces frente a su disfunción, hace previsible un aumento en la demanda de implantación de prótesis de pene en los próximos años, en aquellos pacientes altamente motivados, pero refractarios a los tratamientos menos invasivos.

Palabras clave: Disfunción eréctil. Prótesis de pene. Tratamiento. Impotencia.

\section{ABSTRACT \\ PENILE PROSTHESIS FOR THE TREATMENT OF ERECTILE DYSFUNCTION}

The erectile dysfunction is a pathology that, with different degrees of intensity, affects nearly the $20 \%$ of the spanish adult men. The treatment is usually performed in stages, reserving the penile prosthesis for when other previous treatments have failed. The aim of this work is to evaluate, according to the state of present knowledge, the effectiveness and security of the penile prosthesis for the treatment of the erectile dysfunction. With this purpose 52 articles were selected, observing a 5 years prosthesis survival of 78-91\% and a $3-8 \%$ of surgical complications. Mechanical failures and infection percentages were smaller in the semi-rigid prosthesis that in the inflatable ones, with high levels of postoperative satisfaction in patients as well as in their couples, even greater than in other treatments available at the present time.

The penile prosthesis implantation must be reserved for the organic erectile dysfunction when previous treatments have failed, evaluating the risk-benefit relation and informing the patient of the results that are hoped to be obtained and of the possible complications that can arise.

In view of the great concern of our society with the erectile function and the availability of effective drugs, an increase in the demand of penile prosthesis implantation is predictable in those patients highly motivated, but refractory to the less invasive treatments.

Keywords: Erectile dysfunction. Penile prosthesis. Treatment. Impotence. 
$\mathrm{L}^{2}$ a disfunción eréctil (DE) es definida clásicamente como la "incapacidad persistente para lograr o mantener una rigidez suficiente del pene que permita una actividad sexual satisfactoria”, siendo una patología de alta prevalencia, cifrada según el estudio EDEM $^{1}$ (Epidemiología de la Disfunción Eréctil Masculina) entre el 12 y el $19 \%$ de los varones españoles de edades comprendidas entre los 25 y 70 años. Sin embargo, no hay que olvidar que en grupos seleccionados de pacientes, como por ejemplo la población diabética, la prevalencia de DE podría llegar incluso hasta el 30-50\%. No cabe duda que con el paso de los años las respuestas sexuales se suelen tornar más lentas y menos intensas, pudiéndose hablar de una DE fisiológica debido a la edad. Así, ya en 1948, Kinsey et al. ${ }^{2}$ describieron una prevalencia de disfunción eréctil del 2\% a los 40 años, del $6,7 \%$ a los 50, del $18,4 \%$ a los 60 , del $27 \%$ a los 70 y del $75 \%$ a los 80 . Esta asociación con la edad ha sido atribuida a que el envejecimiento favorece la aparición de enfermedades como la diabetes o la patología vascular, factores de riesgo a su vez para la DE y a la mayor utilización de fármacos potencialmente favorecedores de la misma.

\section{ETIOLOGÍA}

Debido a que la función eréctil requiere una coordinación de factores psicológicos, neurológicos, endocrinos, vasculares y musculares y que cualquier alteración de uno de estos elementos puede contribuir a su disfunción, en la mayoría de las ocasiones la etiología suele ser multifactorial.

En líneas generales, las causas de disfunción eréctil pueden clasificarse en orgánicas y psicológicas, suponiendo las primeras cerca del 75\% del total y siendo las más frecuentes las enfermedades cardiovasculares, endocrinas, neurológicas y prostáticas, las alteraciones estructurales, como la enfermedad de Peyronie, y el consumo de fármacos, tabaco y alcohol. Los factores psicológicos juegan también un papel muy importante y de hecho, casi todos los tipos de DE tienen un componente psicológico en mayor o menor grado, incluso aquellos casos donde existe una causa orgánica conocida.

\section{DIAGNÓSTICo}

El primer paso y tal vez el más importante para llegar al diagnóstico de $\mathrm{DE}$ es la realización de una adecuada historia clínica orientada a determinar su causa más probable, diferenciando entre un origen orgánico o psicógeno. Es importante valorar también a la pareja sexual del paciente, si existe de forma estable, haciendo hincapié en la calidad de dicha relación y si la DE ha afectado a la misma. En todo paciente deberá realizarse una exploración física general, reservando la realización de pruebas complementarias especializadas para el diagnóstico diferencial entre una naturaleza psicógena y orgánica del proceso. Dentro de estas pruebas se incluyen las que persiguen objetivar la presencia de erección espontánea, los estudios de imagen y los neurológicos (Tabla 1).

\section{Tabla 1}

Principales pruebas utilizadas en el diagnóstico de la disfunción eréctil

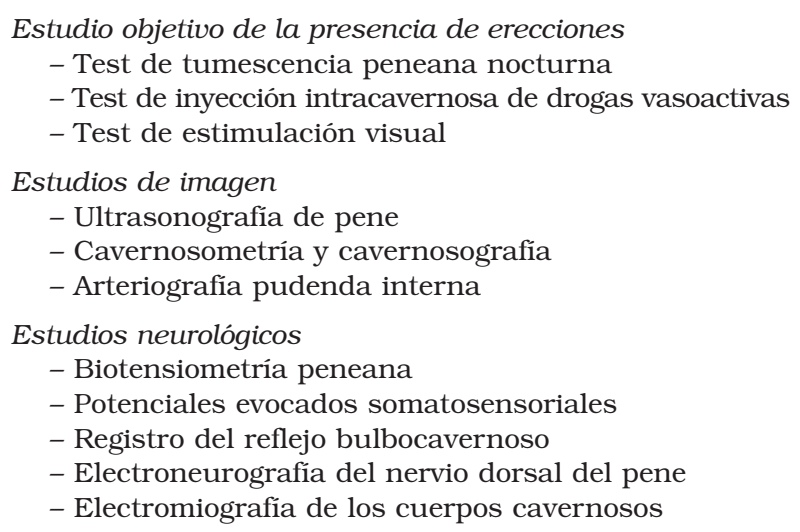

Se han desarrollado diferentes tests con el fin de facilitar y estandarizar el diagnóstico y el grado de DE, siendo los más utilizados, el IIEF (Índice Internacional de Función Eréctil) y el EDITS (Cuestionario de Satisfacción por Tratamiento de Disfunción Eréctil). El primero es un test de gran sensibilidad y especificidad, validado y traducido a nuestro idioma, que consta de 15 preguntas evaluadoras de 5 campos de la función sexual. En ocasiones se utiliza un test abreviado denominado SHIM (Cuestionario de Salud Sexual para Varones) que con 5 preguntas y 6 opciones de respuesta, logra también una gran sensibilidad y especificidad. 


\section{TRATAMIENTO}

Dependerá en primer lugar de la etiología y del grado de severidad de la disfunción, siendo preciso considerar otros factores, como la eficacia y los efectos adversos del mismo, la aceptación por el paciente y su pareja, los efectos psicológicos, su posible reversibilidad y el coste económico. Diferentes autores y foros de consenso ${ }^{3,4}$ recomiendan una instauración escalonada de las diversas modalidades de tratamiento existentes, pudiendo establecerse tres etapas:

\section{Primera etapa}

Incluiría el adecuado control de las posibles enfermedades concomitantes, la revisión de los tratamientos farmacológicos a que está sometido el paciente, pues en muchas ocasiones agravan la propia DE, y la modificación de los hábitos nocivos, como el alcohol o el tabaco, promoviendo además, un estilo de vida saludable. Dentro de esta primera etapa se encuentra también el apoyo o consejo sexual, con el fin de transmitir al paciente y su pareja una serie de pautas de conducta básicas que permitan mejorar la calidad de sus relaciones sexuales.

Por último, y dentro de este primer eslabón, es preciso considerar a los agentes farmacológicos orales, y más concretamente a los inhibidores de la 5-fosfodiesterasa sildenafilo, tadalafilo y vardenafilo. Éstos, desde su introducción, han revolucionado las opciones de tratamiento de la DE, ya que además de ser eficaces son, en general, muy bien tolerados. Otros agentes farmacológicos orales, aunque menos utilizados y eficaces, son la apomorfina sublingual, la yohimbina, la fentolamina y la trazodona.

\section{Segunda etapa}

En este apartado se incluye la terapia intracavernosa con sustancias vasoactivas, como la prostaglandina $\mathrm{E}_{1} \mathrm{o}$ alprostadilo, siendo candidatos aquellos pacientes en los que han fallado o no se pueden utilizar los fármacos orales. Si el alprostadilo en monoterapia no produce el efecto deseado, puede asociarse a otros fármacos como la papaverina o la fentolamina, si bien en España aún no está autorizada la comercialización de dicha asociación. Otras formas de administración del alprostadilo es a través de la uretra, absor- biéndose y pasando a los cuerpos cavernosos, o en forma de gel tópico de aplicación en el glande, no estando tampoco comercializadas.

Por último, y dentro de esta segunda etapa, pueden utilizarse también los dispositivos de vacío consistentes en un cilindro plástico que, colocado en el pene y mediante un mecanismo de succión, produce una entrada de sangre, con la consiguiente erección del mismo. Es un método económico con especial indicación en la población anciana, aunque son poco utilizados en nuestro medio a pesar de que los índices de satisfacción son elevados y las complicaciones mínimas.

\section{Tercera etapa}

Está constituida por las prótesis de pene, clasificándose éstas en dos grupos, semirrígidas e hinchables. Las prótesis semirrígidas se clasifican a su vez en maleables y mecánicas, teniendo el inconveniente de que el pene está siempre en erección, si bien es posible orientarlo en tres posiciones diferentes: recta/elevada para las relaciones sexuales, hacia abajo, para la micción, y posición normal o doblada, a fin de disimularlo con la ropa. Dentro de las maleables, las más utilizadas son las AMS $600^{\circledR}$ y $650^{\circledR}$ (American Medical Systems, Minnetonka, Minnesota) y la Mentor Acu-Form ${ }^{\circledR}$ (Mentor, Santa Bárbara, California), mientras que la única representante del grupo de prótesis semirrígidas mecánicas es la Dura-II ${ }^{\circledR}$ (comercializada en la actualidad por AMS).

Las prótesis hidráulicas o hinchables pueden ser de dos o de tres componentes, ya que las de uno ya no se comercializan en la actualidad. Las de dos componentes consisten en dos cilindros flexibles rellenos de suero salino estéril que se insertan en los cuerpos cavernosos y que están conectados a un dispositivo de bomba, con función también de reservorio, que se instala en el escroto. Para conseguir la erección debe apretarse la bomba escrotal varias veces, lo que transferirá el líquido desde el reservorio a los cilindros. Para su vaciamiento, y conseguir la flacidez del pene, es suficiente con doblar los cilindros hacia abajo durante unos diez segundos, lo que hará que el líquido vuelva al reservorio. Las prótesis

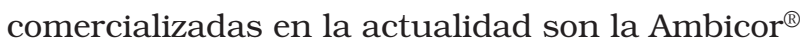
(AMS) y la Excel ${ }^{\circledR}$ (Mentor). 
Las prótesis de tres componentes son las más sofisticadas y están compuestas por dos cilindros que se implantan en los cuerpos cavernosos, siendo independiente la bomba del reservorio, ya que el sistema de bombeo se coloca en el escroto y el reservorio en el peritoneo o en el espacio laterovesical, como lugares más habituales. Dentro de este grupo están la prótesis $\operatorname{Titan}^{\circledR}$ de Mentor y la AMS $700 \mathrm{CX}^{\circledR}, \mathrm{CXM}^{\circledR}$, Ultrex ${ }^{\circledR}$ y Ultrex Plus ${ }^{\circledR}$.

Según los datos del CMBD (Conjunto Mínimo Básico de Datos), durante el periodo de 1997 a 2002 se implantaron en España un total de 1.427 prótesis de pene, de las que 753 fueron semirrígidas y 674 hinchables. Algunas Comunidades presentaron elevadas tasas de implantación, mientras que en otras prácticamente no se utilizó esta tecnología, observándose incluso importantes diferencias entre ellas con respecto a los porcentajes de utilización de prótesis hinchables y semirrígidas, sin que existiesen causas aparentes que los expliquen. Así, nos encontramos con que Cataluña fue la Comunidad con mayor número de prótesis implantadas por 100.000 varones de entre 25 y 70 años de edad, tanto de forma global $(21,13)$ como en el de semirrígidas $(18,98)$, ocupando Galicia el segundo lugar a nivel global $(18,36)$ y el primero en prótesis hinchables $(14,39)$.

El objetivo de este trabajo es evaluar, de acuerdo al estado de conocimiento actual, la efectividad y seguridad de las prótesis de pene en el tratamiento de la disfunción eréctil del varón.

\section{MÉTODOS}

Se realizó una revisión sistemática de la literatura científica existente en las principales bases de datos bibliográficas (Medline y PreMedline, Embase, Health Technology Assesment, Cochrane Database, Dare, Lilacs, etc.), desde 1989 hasta octubre de 2004. Este proceso se completó con una búsqueda general en Internet y con la revisión de las páginas de diferentes organizaciones nacionales e internacionales, sociedades científicas etc., con el fin de localizar otra información que pudiera ser de interés. A continuación se procedió a la lectura de los resúmenes de los artículos, seleccionándose aquellos que cumplian una serie de criterios previamente establecidos en función de los objetivos de este trabajo:
1. Según el diseño del estudio, únicamente se incluyeron revisiones sistemáticas, metaanálisis, ensayos clínicos aleatorizados o no, estudios de cohortes, estudios de casos y controles y estudios descriptivos de series de casos, excluyéndose los estudios descriptivos de un solo caso, las revisiones narrativas y las cartas al editor, editoriales, comentarios y comunicaciones a congresos.

2. Según el idioma, todos aquellos publicados en los idiomas castellano, inglés, francés, portugués e italiano.

3. Según la población de estudio, los realizados en varones, independientemente de la edad, sometidos a una intervención de implante primario o reemplazo de prótesis de pene.

4. Según la patología, solamente los estudios que incluyesen pacientes con $\mathrm{DE}$ de carácter orgánico.

5. Según la medición de resultados, se incluyeron aquellos artículos que referian datos relativos a eficacia/efectividad de la prótesis implantada, de supervivencia de la misma a largo plazo, de cambios en la función sexual, satisfacción y calidad de vida, de incidencia y/o tipos de complicaciones postoperatorias, de fallo de la prótesis y de análisis de costes.

Por último, se procedió a una lectura crítica de los artículos seleccionados, revisándose también la bibliografia citada en los mismos y, mediante un formulario diseñado específicamente, al análisis y extracción de datos para la evaluación de los mismos. La calidad de los artículos se valoró siguiendo la escala de Jovell y Navarro-Rubio ${ }^{5}$, en la que se establece una jerarquía de la evidencia científica.

\section{RESULTADOS}

El número de artículos seleccionados fue de $52^{6-57}$, todos ellos series clínicas no controladas, sin que se encontrasen estudios de mayor calidad metodológica que analizasen aspectos de esta tecnologia.

El número de pacientes evaluados en los diferentes artículos fue variable, existiendo 34 con un tamaño muestral menor de 200, 15 con entre 201 y 500 pacientes y 3 que incluyeron más de 1.000. La media de edad en el conjunto de estudios fue de 57,3 \pm 4 años y con un periodo de disfunción eréctil previo a la inserción de la prótesis 
de 3,79 $\pm 0,83$ años. Las principales etiologías fueron las de causa vascular $(55,25 \%$ de los casos), diabetes $(21,08 \%)$, enfermedad de Peyronie $(8,96 \%)$, cirugía radical $(8,70 \%)$ y enfermedades neurológicas $(6,1 \%)$. Con respecto al abordaje quirúrgico, las vías más utilizadas fueron la penoescrotal y la infrapúbica.

\section{Resultados de efectividad}

La evaluación de la efectividad se realizó en base al análisis de la función de supervivencia de la prótesis y al porcentaje de éxito de la misma a la hora de proporcionar una erección adecuada para el coito.

Con respecto al primer punto, los artículos publicados más recientemente reflejan supervivencias libres de complicación a los 5 años del $78 \%{ }^{52}$ para las prótesis AMS 700 Ultrex $^{\circledR}$, de entre el $70-91 \%^{14,33,46,47}$ para las prótesis AMS $700 \mathrm{CX}^{\circledR} / \mathrm{CXM}^{\circledR}$ y del $97 \%{ }^{51}$ a los 6 años para la prótesis Ambicor ${ }^{\circledR}$, siendo estos porcentajes incluso superiores si sólo se tiene en cuenta la supervivencia libre de fallo mecánico.

A la hora de valorar la consecución de una erección adecuada para el coito deben tenerse en cuenta los bajos porcentajes de respuesta a los cuestionarios y otros sesgos de diseño, si bien los diferentes artículos refieren porcentajes de éxito entre el 70 y el $90 \%$ de los casos.

\section{Resultados de seguridad}

Los parámetros utilizados para evaluar la seguridad de las prótesis de pene se agruparon en cuatro apartados:

Complicaciones quirúrgicas: todas las derivadas del proceso quirúrgico, tanto las intraoperatorias como las postquirúrgicas tempranas y tardías.

Complicaciones mecánicas o fallos de los dispositivos: cualquier evento que hizo que un dispositivo pasase a ser no funcional de tal manera que fuese necesario una intervención para que volviese a ser utilizable.

Complicaciones infecciosas: sólo se tuvieron en cuenta las infecciones de prótesis, omitiéndose las superficiales de la herida quirúrgica.

Reemplazo o extracción de la prótesis: independientemente de la causa que lo provocó, sin que se incluyesen aquí aquellos casos en los que sólo se realizó una revisión quirúrgica.
En la Tabla 2 se presentan los porcentajes acumulados de las diferentes complicaciones agrupadas por tipo de prótesis empleada.

Como era de esperar, el tiempo transcurrido entre la implantación de la prótesis y la aparición de complicaciones sigue una relación directa, describiendo Randrud ${ }^{25}$ una correlación significativa $(\mathrm{p}=0,01)$ entre la aparición del fallo mecánico y la duración del seguimiento. También Choi et al. ${ }^{50}$ observaron a los 3 años un $55 \%$ de fallos mecánicos, a los 5 años un $85 \%$ y a los 6 años, el $100 \%$ de los mismos. Por término medio, las complicaciones postoperatorias aparecieron poco tiempo después de la intervención (media de $2,5^{20}$ y 4,8 meses $^{37}$, mientras que las infecciones protésicas lo hicieron entre $4^{25}$ y $7^{51}$ meses. Con respecto a los fallos mecánicos, los estudios que analizaron este parámetro hallaron medias de 19,7 meses $^{25}, 22,3$ meses $^{51}$, 32,1 meses $^{37}$ y 40 meses $^{36}$.

Con respecto a las infecciones, destacar el estudio de Wilson et al. ${ }^{26}$, que aunque no incluido en la tabla anterior debido a la utilización de once tipos de prótesis diferentes y estar centrado exclusivamente en el análisis de la infección como complicación, fue llevado a cabo en un importante número de pacientes $(1.142$ pacientes receptores de 1.337 prótesis primarias o secundarias) y con un tiempo de seguimiento de 7 años. Los autores encontraron un porcentaje global de infección del 5\%, siendo menor (3\%) en implantes primarios que en prótesis reimplantadas (10\%). De las infecciones producidas en implantes primarios, el $50 \%$ se produjeron en pacientes con tratamiento crónico de esteroides, el 9\% en lesionados medulares, el 3\% en pacientes diabéticos y el resto en otros pacientes. Con respecto a los implantes secundarios, el $18 \%$ de las infecciones ocurrieron en pacientes diabéticos, el $8 \%$ en primer reemplazo de no diabéticos, el $12 \%$ en no diabéticos con reemplazos múltiples y el $10 \%$ en pacientes con reimplante diferido.

Es preciso mostrar también tres estudios comparativos entre prótesis antiguas y modelos posteriores mejorados (Tabla 3). Así, Nickas ${ }^{20}$ comparó prótesis AMS 700 CX" con prótesis de los modelos PP, PPL, PPR, PPS y PPT, existiendo una importante diferencia en el número de com- 
Tabla 2

Porcentajes acumulados de complicaciones quirúrgicas, mecánicas, infecciosas y de reemplazos por los diferentes tipos de prótesis. Elaboración propia.

\begin{tabular}{|c|c|c|c|c|}
\hline $\begin{array}{l}\text { Tipo de prótesis } \\
\text { (n) }\end{array}$ & $\begin{array}{l}\text { Complicaciones } \\
\text { Quirúrgicas }\end{array}$ & $\begin{array}{l}\text { Complicaciones } \\
\text { Mecánicas }\end{array}$ & Infecciones & $\begin{array}{l}\text { Reemplazos } \\
\text { o extracciones }\end{array}$ \\
\hline $\begin{array}{l}\text { AMS 600 } \\
\quad(231)\end{array}$ & $\begin{array}{c}3,3 \% \\
(2 \mathrm{de} 61)\end{array}$ & $\begin{array}{c}0 \% \\
(0 \text { de } 231)\end{array}$ & $\mathrm{NE}$ & $\mathrm{NE}$ \\
\hline $\begin{array}{l}\text { Duraphase }^{\circledR} \\
\text { (112) }\end{array}$ & $\begin{array}{c}4,5 \% \\
(4 \mathrm{de} 89)\end{array}$ & $\begin{array}{c}8,03 \% \\
(9 \text { de } 112)\end{array}$ & $\begin{array}{l}2,25 \% \\
(2 \mathrm{de} 89)\end{array}$ & $\begin{array}{l}10,11 \% \\
(9 \text { de } 89)\end{array}$ \\
\hline $\begin{array}{l}\text { Dura II }^{\circledR} \\
(290)\end{array}$ & $\begin{array}{c}10,34 \% \\
(30 \text { de } 290)\end{array}$ & $\begin{array}{c}0 \% \\
\text { (0 de } 290)\end{array}$ & $\begin{array}{c}1 \% \\
(3 \text { de } 290)\end{array}$ & $\begin{array}{c}6,21 \% \\
(18 \text { de } 290)\end{array}$ \\
\hline $\begin{array}{c}\text { Total Semirrigidas } \\
\text { (633) }\end{array}$ & $\begin{array}{c}8,18 \% \\
(36 \text { de } 440)\end{array}$ & $\begin{array}{c}1,4 \% \\
\text { (9 de } 633 \text { ) }\end{array}$ & $\begin{array}{c}1,3 \% \\
\text { (5 de } 379 \text { ) }\end{array}$ & $\begin{array}{c}7,1 \% \\
(27 \text { de } 379)\end{array}$ \\
\hline $\begin{array}{l}\text { Hydroflex }^{\circledR} \\
\text { (325) }\end{array}$ & $\mathrm{NE}$ & $\begin{array}{c}18,15 \% \\
\text { (59 de } 325)\end{array}$ & $\begin{array}{c}2,4 \% \\
(6 \text { de } 253)\end{array}$ & $\begin{array}{c}33,3 \% \\
\text { (89 de } 267)\end{array}$ \\
\hline $\begin{array}{l}\text { Dynaflex }^{\circledR} \\
(461)\end{array}$ & $\begin{array}{c}6,56 \% \\
(12 \mathrm{de} 183)\end{array}$ & $\begin{array}{c}8,89 \% \\
(41 \mathrm{de} 461)\end{array}$ & $\begin{array}{c}4,29 \% \\
\text { (14 de } 326)\end{array}$ & $\begin{array}{c}17,86 \% \\
(40 \text { de } 224)\end{array}$ \\
\hline $\begin{array}{c}\text { Total Hidr. } 1 \text { comp. } \\
(786)\end{array}$ & $\begin{array}{c}6,56 \% \\
(12 \text { de } 183)\end{array}$ & $\begin{array}{c}12,72 \% \\
(100 \text { de } 786)\end{array}$ & $\begin{array}{c}3,45 \% \\
(20 \text { de } 579)\end{array}$ & $\begin{array}{c}26,27 \% \\
(129 \text { de } 491)\end{array}$ \\
\hline $\begin{array}{c}\text { GFS }^{\circledR} / \text { GFS Mark } \mathrm{II}^{\circledR} \\
(311)\end{array}$ & $\begin{array}{c}4,82 \% \\
\text { (11 de } 228)\end{array}$ & $\begin{array}{c}14,8 \% \\
(46 \text { de } 311)\end{array}$ & $\begin{array}{c}4,07 \% \\
(7 \text { de } 172)\end{array}$ & $\begin{array}{c}8,14 \% \\
(14 \text { de } 172)\end{array}$ \\
\hline $\begin{array}{c}\text { Ambicor }^{\circledR} \\
(139)\end{array}$ & $\begin{array}{c}0,8 \% \\
(1 \mathrm{de} 131)\end{array}$ & $\begin{array}{c}2,16 \% \\
(3 \text { de } 139)\end{array}$ & $\begin{array}{c}4,6 \% \\
(6 \text { de } 131)\end{array}$ & $\begin{array}{c}3,8 \% \\
(5 \text { de } 131)\end{array}$ \\
\hline $\begin{array}{c}\text { Total Hidr. } 2 \text { comp. } \\
(450)\end{array}$ & $\begin{array}{c}3,34 \% \\
(12 \text { de } 359)\end{array}$ & $\begin{array}{c}10,89 \% \\
(49 \text { de } 450)\end{array}$ & $\begin{array}{c}4,29 \% \\
(13 \text { de } 303)\end{array}$ & $\begin{array}{c}6,27 \% \\
(19 \text { de } 303)\end{array}$ \\
\hline $\begin{array}{c}\text { AMS serie } 700^{\circledR} \\
(224)\end{array}$ & $\begin{array}{c}11,63 \% \\
(25 \text { de } 215)\end{array}$ & $\begin{array}{c}39,73 \% \\
\text { (89 de } 224)\end{array}$ & $\begin{array}{c}1,4 \% \\
(3 \mathrm{de} 215)\end{array}$ & $\mathrm{NE}$ \\
\hline $\begin{array}{c}\text { AMS } 700 \mathrm{CX}^{\circledR} / \mathrm{CXM}^{\circledR} \\
(1794)\end{array}$ & $\begin{array}{c}8,06 \% \\
(64 \text { de } 794)\end{array}$ & $\begin{array}{c}10,42 \% \\
(187 \text { de } 1794)\end{array}$ & $\begin{array}{c}3,70 \% \\
\text { (52 de } 1404)\end{array}$ & $\begin{array}{c}13,06 \% \\
(64 \text { de } 490)\end{array}$ \\
\hline $\begin{array}{c}\text { AMS } 700 \text { Ultrex }^{\circledR} / \text { Ultrex } \\
\text { Plus }^{\circledR}(766)\end{array}$ & $\begin{array}{c}5,58 \% \\
(21 \text { de } 376)\end{array}$ & $\begin{array}{c}12,27 \% \\
(94 \text { de } 766)\end{array}$ & $\begin{array}{c}4,73 \% \\
(23 \text { de } 486)\end{array}$ & $\begin{array}{c}9,34 \% \\
(45 \text { de } 482)\end{array}$ \\
\hline $\begin{array}{c}\text { Mentor Alpha I }{ }^{\circledR} \\
\text { (3303) (datos globales) }\end{array}$ & $\begin{array}{c}6,64 \% \\
(160 \text { de } 2410)\end{array}$ & $\begin{array}{c}6,18 \% \\
(204 \text { de } 3303)\end{array}$ & $\begin{array}{c}3,29 \% \\
(103 \mathrm{de} 3130)\end{array}$ & $\begin{array}{c}5,92 \% \\
(97 \text { de } 1637)\end{array}$ \\
\hline $\begin{array}{c}\text { Total Hidr. } 3 \text { comp. } \\
(6087)\end{array}$ & $\begin{array}{c}7,11 \% \\
(270 \text { de } 3795)\end{array}$ & $\begin{array}{c}9,43 \% \\
(574 \text { de } 6087)\end{array}$ & $\begin{array}{c}3,46 \% \\
(181 \text { de } 5235)\end{array}$ & $\begin{array}{c}7,90 \% \\
\text { (206 de 2609) }\end{array}$ \\
\hline
\end{tabular}

(n): tamaño muestral. NE: no especificado.

plicaciones de índole mecánica, disminuyendo las mismas desde el 40,9\% de los modelos antiguos a un $5,45 \%$ en aquellas que sufrieron la modificación en su diseño. Milbank et al. ${ }^{52}$ compararon la prótesis AMS 700 Ultrex $^{\circledR}$ antes y después de la modificación en el diseño de la misma realizada en 1993, reduciéndose el número de complicaciones de índole mecánica desde el
$44,7 \%$ al $7,7 \%$ en las modificadas. De la misma forma, Wilson et al. ${ }^{45}$ realizaron un estudio comparativo entre prótesis Mentor Alpha ${ }^{\circledR}$ fabricadas antes y después de noviembre de 1992, observando una reducción en las complicaciones mecánicas desde el $21,7 \%$ en las prótesis anteriores a 1992 a un $2,8 \%$ en aquellas sometidas a modificaciones en su diseño. 
Tabla 3

Complicaciones mecánicas en prótesis AMS serie $700^{\circledR}$, AMS 700 Ultrex $^{\circledR}$ y Mentor ${ }^{\circledR}$, antes y después de las modificaciones sufridas en sus diseños. Elaboración propia

\begin{tabular}{|c|c|c|c|}
\hline Prótesis & AMS serie $700^{\circledR 20}$ & AMS 700 Ultrex ${ }^{\circledR 52}$ & Mentor ${ }^{\circledR 45}$ \\
\hline Modelos antiguos & 88 de $215(40,9 \%)$ & 38 de $85(44,7 \%)$ & 89 de $410(21,7 \%)$ \\
\hline Modelos reformados & 3 de 55 (5,45\%) & 4 de $52(7,7 \%)$ & 27 de $971(2,8 \%)$ \\
\hline
\end{tabular}

nico en los que hayan fracasado otros tratamientos previos, cuando éstos sean considerados inaceptables por el paciente o en los casos de malformaciones complejas del pene.

Es importante que la recomendación de una prótesis de pene se realice siempre de forma individual, teniendo en cuenta la relación riesgo/beneficio y estando informado el paciente de los resultados que se esperan conseguir en cuanto a capacidad de erección y de las posibles complicaciones que puedan surgir. El paciente deberá conocer también las diferentes opciones protésicas disponibles en la actualidad, debiendo el médico asesorarlo en función de sus hábitos y su destreza manual. Por último, el paciente debe ser consciente de la irreversibilidad del procedimiento al que se va a someter, dado que la implantación de una prótesis conlleva el empuje del tejido esponjoso hacia la periferia para dejar espacio a los cilindros, por lo que si éstos son retirados posteriormente por cualquier razón, el espacio será ocupado por tejido cicatricial que no responderá adecuadamente a otros tratamientos como los fármacos vasoactivos intracavernosos o los dispositivos de vacío.

\section{EFECTIVIDAD DE LAS PRÓTESIS DE PENE}

La prótesis de pene ideal sería aquella que lograse un estado de flacidez y erección similar al estado natural y con una supervivencia del 100\% tras un largo periodo de tiempo. Los nuevos diseños de prótesis, eliminando o mejorando aquellos puntos de las mismas que más frecuentemente producian fallos, unido a la mayor experiencia de los cirujanos en el manejo de estos dispositivos, ha hecho que en la actualidad se alcancen altas cotas de efectividad, como puede comprobarse al analizar los artículos publicados más recientemente. Sin embargo, estos estudios no se prolongaron más allá de los 5 o 6 años, por lo que se desconoce cual es el tiempo de vida máximo de una prótesis y con qué frecuencia se precisa su recambio. Con respecto al porcentaje de éxito a la hora de proporcionar una erección adecuada para el coito, es preciso tener en cuenta los bajos porcentajes de respuesta obtenida en muchos 
casos y otros sesgos de diseño, por lo que los buenos resultados obtenidos deben considerarse con precaución.

\section{SEGURIDAD DE LAS PRÓTESIS DE PENE}

Complicaciones quirúrgicas y reemplazos de prótesis

En líneas generales, las complicaciones quirúrgicas relativas a la implantación de prótesis aparecen entre el 3 y el 8\% de los casos, situándose el porcentaje de reemplazo o retirada de prótesis debido a cualquier complicación en el 7\%. Es preciso tener en cuenta que siempre que se pone en marcha cualquier técnica quirúrgica hay que considerar un cierto período de entrenamiento $\mathrm{y}$ perfeccionamiento técnico denominado “curva de aprendizaje", en el que los resultados y complicaciones pueden no ser los esperados, siendo más o menos prolongado y requiriendo un mayor número de casos, en función de la dificultad del procedimiento a ejecutar.

Dentro de las diferentes complicaciones quirúrgicas, merece especial mención la erosión o abrasión del tejido próximo a la prótesis, ya que habitualmente requiere la explantación de la misma. Los porcentajes de erosión descritos en la literatura son variables, con porcentajes entre el $1 \%$ y el 3\%. Otra complicación importante es la migración de algún componente de la prótesis, siendo la más común la de la bomba desde el escroto, que afecta no sólo al aspecto estético, sino que puede dificultar también su accionamiento y la propia relación sexual al chocar con la base del pene. Los porcentajes citados en la literatura respecto a la migración de la bomba o del reservorio varían de forma importante, entre el $0,8 \%$ y el $5,4 \%$.

\section{Complicaciones mecánicas}

Los fallos mecánicos aparecen en todos los tipos de prótesis, aunque con un enorme grado de variabilidad, siendo los más frecuentes, las fugas de líquido a través del reservorio, del tubo o de las conexiones y los propios fallos de la bomba. En líneas generales, las prótesis con menor número de este tipo de complicaciones son las semirrígidas, debido a la sencillez de su diseño, mientras que las prótesis hinchables ofrecen una flacidez y erección más parecidas al estado natural, a costa de un mayor riesgo de fallo mecánico y por ende de reintervención. Destacar las diferencias entre los resultados obtenidos por los modelos actuales y los de las prótesis más antiguas, con disminuciones en el porcentaje de complicaciones desde el 40-45\% al 5-8\%, debido a las mejoras obtenidas en el diseño y en los materiales.

\section{Complicaciones infecciosas}

La infección es seguramente la complicación más importante de la cirugía de implante de prótesis de pene, ya que requiere habitualmente una reintervención y con frecuencia la retirada de la prótesis. En esta revisión, con un total de 6.500 pacientes tratados en los diferentes estudios, el porcentaje encontrado fue del 1,3\% para las prótesis semirrígidas y alrededor del 3,5\% para las hinchables. Entre los factores a tener en cuenta destaca si el implante es primario o se trata de un reimplante, ya que en el primer caso la infección es del 3\%, mientras que en el segundo oscila entre el 10 y el 18\%. Otros factores que pueden incidir son el tratamiento crónico con esteroides, la diabetes y la lesión medular.

Ante una infección protésica, lo habitual es extraer todos sus componentes, esperando de dos a seis meses antes de insertar una nueva. Sin embargo, y para evitar la fibrosis de los cuerpos cavernosos que se produce tras la retirada y que dificulta en gran manera el reimplante, se recurre en ocasiones a la cirugía de rescate con reimplante inmediato, o al rescate diferido, que se realiza al cabo de unas 72 horas.

En la actualidad existe en el mercado un sistema de tratamiento de la superficie de las prótesis hinchables, con una formulación antibiótica a base de minociclina y rifampicina, que parece reducir la tasa de infección protésica. Sin embargo, algunos autores advierten de los potenciales riesgos de estos procedimientos antimicrobianos, ya que pudieran producir alergia y resistencia antibióticas.

\section{SATISFACCIÓN}

La satisfacción con un tratamiento no es un resultado fácilmente medible, sino que va ligada a las expectativas que el paciente o su pareja tenían del mismo y está relacionada con factores 
tan diversos como las complicaciones postoperatorias, resultados cosméticos, función del implante, facilidad de uso y aceptación por parte de la pareja. Un buen número de estudios indican que las prótesis de pene proporcionan, tanto entre los pacientes como entre sus parejas, unos altos niveles de satisfacción postoperatoria, mayores incluso que el resto de tratamientos disponibles en la actualidad para la DE. Sin embargo, es preciso tener en cuenta que la mayoría de los estudios de satisfacción fueron retrospectivos, que algunos incluyeron sólo al paciente y que en la mayoría de las ocasiones se utilizaron cuestionarios no validados.

\section{OTRAS CONSIDERACIONES}

En apartados anteriores hemos visto la existencia de Comunidades con elevadas tasas de implantación de prótesis de pene junto a otras en las que prácticamente no se utilizan estos dispositivos, observándose incluso, importantes diferencias en los porcentajes de utilización de las diferentes modalidades de prótesis existentes, sin que existan causas aparentes que los expliquen. Sería por ello necesario establecer, de forma consensuada, una serie de criterios que permitiesen la adecuada selección y priorización de los pacientes susceptibles de esta tecnología, teniendo en cuenta factores como el grado de disfunción eréctil, los tratamientos previos utilizados, la motivación, la edad, las enfermedades asociadas $\mathrm{y}$ todos aquellos otros factores que se consideren para, de esta manera, evitar la enorme variabilidad geográfica observada en este procedimiento, garantizando la equidad terapéutica y la calidad de los servicios prestados.

Con respecto a la previsión de utilización de esta tecnología, es preciso tener en cuenta que en los últimos años, la sociedad ha sufrido un cambio en la percepción de la sexualidad humana con una mayor preocupación en la función eréctil que ha dado como resultado un notable incremento en el número de hombres que solicitan tratamiento de su disfunción. Además, la actual disponibilidad de una farmacoterapia eficaz para su tratamiento, unido a que los pacientes prefieren métodos poco invasivos a pesar de su menor efectividad, ha hecho que los agentes farmacológicos orales del tipo de los inhibidores de la 5-fos- fodiesterasa se hayan generalizado entre la población. Sin embargo, este mayor consumo de fármacos está provocando que a los pacientes que fracasan en la terapia farmacológica se les ofrezcan tratamientos de segunda línea, como los dispositivos de vacío o las inyecciones intracavernosas, siendo probable que, en el futuro, se incremente la demanda de implantación de prótesis de pene para aquellos pacientes altamente motivados pero refractarios a los tratamientos de primera y segunda línea.

Agradecimientos. A los Dres. Antonio Marqués Queimadelos y Venancio Chantada Abal, por su amabilidad en la lectura del manuscrito original y en sus aportaciones al mismo.

\section{REFERENCIAS}

1. Martín-Morales A, Sánchez-Cruz JJ, Saenz de Tejada I, Rodríguez-Vela L, Jiménez-Cruz JF, Burgos-Rodríguez R. Prevalence and independent risk factors for erectile dysfunction in Spain: results of the "Epidemiologia de la Disfuncion Erectil Masculina” Study. J Urol 2001 Aug; 166 (2):569-574; discussion 574-575.

2. Kinsey AC, Pomeroy WB, Martin CE. Sexual behavior in the human male. Philadelphia, WB Saunders, 1948.

3. Foro para la Salud del Hombre en Disfunción Eréctil. Documento de Consenso sobre Disfunción Eréctil. (Consultado 11/01/2005). Disponible en http://www. asesa.org/dominios/asesa.org/ Documento_de Consenso_ sobre_ DE.pdf.

4. Montague DK, Barada JH, Belker AM, Levine LA, Nadig PW, Roehrborn CG, et al. Clinical guidelines panel on erectile dysfunction: summary report on the treatment of organic erectile dysfunction. The American Urological Association. J Urol 1996 Dec;156(6):2007-2011.

5. Jovell AJ, Navarro-Rubio MD. Evaluation of scientific evidence. Med Clin (Barc) 1995 Dec 2;105(19):740-743.

6. Villavicencio H, Parra L, Ruiz Castane E. Surgical treatment with prosthesis implantation in 100 patients with erectile sexual dysfunction. Actas Urol Esp 1989 Nov-Dec; 13(6):420-424.

7. Engel RM, Fein RL. Mentor GFS inflatable prosthesis. Urology 1990 May;35(5):405-406.

8. Hrebinko R, Bahnson RR, Schwentker FN, O'Donnell WF. Early experience with the DuraPhase penile prosthesis. J Urol 1990 Jan;143(1):60-61.

9. Knoll LD, Furlow WL, Motley RC. Clinical experience implanting an inflatable penile prosthesis with controlledexpansion cylinder. Urology 1990 Dec;36(6):502-504.

10. Mulcahy JJ, Krane RJ, Lloyd LK, Edson M, Siroky MB. Duraphase penile prosthesis-results of clinical trials in 63 patients. J Urol 1990 Mar;143(3):518-519.

11. Whalen RK, Merrill DC. Patient satisfaction with Mentor inflatable penile prosthesis. Urology 1991 Jun;37(6):531539.

12. McLaren RH, Barrett DM. Patient and partner satisfaction with the AMS 700 penile prosthesis. J Urol 1992 Jan;147 (1):62-65. 
13. Goldstein I, Bertero EB, Kaufman JM, Witten FR, Hubbard JG, Fitch WP, et al. Early experience with the first pre-connected 3-piece inflatable penile prosthesis: the Mentor Alpha-1. J Urol 1993 Dec;150(6):1814-1818.

14. Quesada ET, Light JK. The AMS 700 inflatable penile prosthesis: long-term experience with the controlled expansion cylinders. J Urol 1993 Jan;149(1):46-48.

15. Randrup E, Wilson S, Mobley D, Suarez G, Mekras G, Baum N. Clinical experience with Mentor Alpha I inflatable penile prosthesis. Report on 333 cases. Urology 1993 Sep;42(3):305-308.

16. Riehmann M, Gasser TC, Bruskewitz RC. The Hydroflex penile prosthesis: a test case for the introduction of new urological technology. J Urol 1993 May;149(5 Pt 2):13041307.

17. Rossi D, Rattier C, Belicar M, Bladou F, Hermanowicz M, Coulange $\mathrm{C}$, et al. Les prothèses de verge gonflables. Expérience à propos de 58 implantations. Prog Urol 1993 Oct;3(5):796-802.

18. Choi HK, Cho IR, Xin ZC. Ten years of experience with various penile prosthesis in Korean. Yonsei Med J 1994 Jun;35(2):209-217.

19. Fein RL. GFS Mark II inflatable penile prosthesis: fouryear clinical study. Urology $1994 \mathrm{Feb}$;3(2):209-213.

20. Nickas ME, Kessler R, Kabalin JN. Long-term experience with controlled expansion cylinders in the AMS 700CX inflatable penile prosthesis and comparison with earlier versions of the Scott inflatable penile prosthesis. Urology 1994 Sep;44(3):400-403.

21. Pereira Arias JG, Escobal Tamayo V, Marana Fernandez MT, Astobieta Odriozola A, Bernuy Malfaz C. Penile prosthetic implant in the treatment of impotence: our experience. Arch Esp Urol 1994 Sep;47(7):703-8.

22. George VK, Erkhan S, Dhabuwala CB. Follow-up with Mentor two-piece inflatable penile prosthesis. Int J Impot Res 1995 Mar;7(1):17-21.

23. Kim SC. Mechanical reliability of AMS hydraulic penile prostheses. J Korean Med Sci 1995 Dec;10(6):422-425.

24. Lewis RW. Long-term results of penile prosthetic implants. Urol Clin North Am 1995 Nov;22(4):847-856.

25. Randrup ER. Clinical experience with 180 inflatable penile prostheses. South Med J 1995 Jan;88(1):47-51.

26. Wilson SK, Delk JR 2nd. Inflatable penile implant infection: predisposing factors and treatment suggestions. J Urol 1995 Mar;153(3 Pt 1):659-661.

27. Anafarta K, Safak M, Beduk Y, Baltaci S, Aydos K. Clinical experience with inflatable and malleable penile implants in 104 patients. Urol Int 1996;56(2):100-104.

28. Garber BB. Inflatable penile prosthesis: results of 150 cases. Br J Urol 1996 Dec;78(6):933-935.

29. Kearse WS Jr, Sago AL, Peretsman SJ, Bolton JO, Holcomb RG, Reddy PK, et al. Report of a multicenter clinical evaluation of the Dura-II penile prosthesis. J Urol 1996 May; 155(5): 1613-1616.

30. Rossello Barbara M. Penile prosthesis. Indications, classification, technic, complications, and safety. Arch Esp Urol 1996 Apr;49(3):294-302.

31. Wilson SK, Cleves M, Delk JR 2nd. Long-term results with Hydroflex and Dynaflex penile prostheses: device survival comparison to multicomponent inflatables. J Urol 1996 May; 155(5): 1621-1623.

32. Burns-Cox N, Burston A, Gingell JC. Fifteen years experience of penile prosthesis insertion. Int J Impot Res 1997 Dec;9(4):211-216.
33. Daitch JA, Angermeier KW, Lakin MM, Ingleright $\mathrm{BJ}$, Montague DK. Long-term mechanical reliability of AMS 700 series inflatable penile prostheses: comparison of CX/CXM and Ultrex cylinders. J Urol 1997 Oct;158(4): 1400-1402.

34. Goldstein I, Newman L, Baum N, Brooks M, Chaikin L, Goldberg K, et al. Safety and efficacy outcome of Mentor Alpha-1 inflatable penile prosthesis implantation for impotence treatment. J Urol 1997 Mar;157(3):833-839.

35. Holloway FB, Farah RN. Intermediate term assessment of the reliability, function and patient satisfaction with the AMS700 Ultrex penile prosthesis. J Urol 1997 May;157(5): 1687-1691.

36. Kabalin JN, Kuo JC. Long-term followup of and patient satisfaction with the Dynaflex self-contained inflatable penile prosthesis. J Urol 1997 Aug; 158(2):456-459.

37. Nukui F, Okamoto S, Nagata M, Kurokawa J, Fukui J. Complications and reimplantation of penile implants. Int $\mathrm{J}$ Urol 1997 Jan;4(1):52-54.

38. Rogers E, Murphy DM. Use of the AMS inflatable penile prosthesis in the management of erectile impotence. Ir Med J 1997 Apr-May;90(3):105-106.

39. Rossi D, Ayuso D, Rattier C, Bladou F, Hermanowicz M, Serment. Clinical experience with 80 inflatable penile prostheses. Eur Urol 1997;31(3):335-338.

40. Anafarta K, Yaman O, Aydos K. Clinical experience with Dynaflex penile prostheses in 120 patients. Urology 1998 Dec;52(6): 1098-1100.

41. Dubocq F, Tefilli MV, Gheiler EL, Li H, Dhabuwala CB. Long-term mechanical reliability of multicomponent inflatable penile prosthesis: comparison of device survival. Urology 1998 Aug;52(2):277-281.

42. Govier FE, Gibbons RP, Correa RJ, Pritchett TR, KramerLevien D. Mechanical reliability, surgical complications, and patient and partner satisfaction of the modern threepiece inflatable penile prosthesis. Urology 1998 Aug;52(2): 282-286.

43. Liberman SN, Gomella LG, Hirsch IH. Experience with the Ultrex and Ultrex Plus inflatable penile prosthesis: new implantation techniques and surgical outcome. Int $\mathrm{J}$ Impot Res 1998 Sep;10(3):175-179.

44. Porena M, Mearini L, Mearini E, Marzi M, Zucchi A. Penile prosthesis implantation and couple's satisfaction. Urol Int 1999;63(3): 185-187.

45. Wilson SK, Cleves MA, Delk JR 2nd. Comparison of mechanical reliability of original and enhanced Mentor Alpha I penile prosthesis. J Urol 1999 Sep;162(3 Pt 1):715-718.

46. Carson CC, Mulcahy JJ, Govier FE. Efficacy, safety and patient satisfaction outcomes of the AMS 700CX inflatable penile prosthesis: results of a long-term multicenter study. AMS 700CX Study Group. J Urol 2000 Aug;164(2):376380.

47. Chiang HS, Wu CC, Wen TC. 10 years of experience with penile prosthesis implantation in Taiwanese patients. J Urol $2000 \mathrm{Feb}$; 163(2):476-480.

48. Cruz Guerra NA, Allona Almagro A, Clemente Ramos L, Navio Nino S, Saenz de Tejada y Gorman I, Linares Quevedo A, et al. Replacement of prosthesis of the penis. Actas Urol Esp 2000 Oct;24(9):728-734.

49. Montorsi F, Rigatti P, Carmignani G, Corbu C, Campo B, Ordesi G, et al. AMS three-piece inflatable implants for erectile dysfunction: a long-term multi-institutional study in 200 consecutive patients. Eur Urol 2000 Jan;37(1): 5055. 
50. Choi YD, Jin Choi Y, Hwan Kim J, Ki Choi H. Mechanical reliability of the AMS 700CXM inflatable penile prosthesis for the treatment of male erectile dysfunction. J Urol 2001 Mar;165(3):822-824.

51. Levine LA, Estrada CR, Morgentaler A. Mechanical reliability and safety of, and patient satisfaction with the Ambicor inflatable penile prosthesis: results of a 2 center study. J Urol 2001 Sep;166(3):932-937.

52. Milbank AJ, Montague DK, Angermeier KW, Lakin MM, Worley SE. Mechanical failure of the American Medical Systems Ultrex inflatable penile prosthesis: before and after 1993 structural modification. J Urol 2002 Jun;167 (6):2502-2506.

53. Rodríguez Tolra J, Franco Miranda E, Arbelaez Arango S, Trilla Herrera E, Serrallach Mila N. Our experience with the implant of single component integrated prostheses (Hydroflex-Dynaflex). Actas Urol Esp 2002 Apr;26(4):261265.

54. Ferguson $\mathrm{KH}$, Cespedes RD. Prospective long-term results and quality-of-life assessment after Dura-II penile prosthesis placement. Urology 2003 Feb;61(2):437-441.

55. Mulhall JP, Ahmed A, Branch J, Parker M. Serial assessment of efficacy and satisfaction profiles following penile prosthesis surgery. J Urol 2003 Apr;169(4):1429-1433.
56. Rajpurkar A, Dhabuwala CB. Comparison of satisfaction rates and erectile function in patients treated with sildenafil, intracavernous prostaglandin E1 and penile implant surgery for erectile dysfunction in urology practice. J Urol 2003 Jul;170(1): 159-163.

57. Rodríguez Faba O, Martín Benito JL, Escaf Bramada S., Rodríguez Martínez J.J., Jalón Monzon A, Regadera Sejas J. Tratamiento de la disfunción eréctil mediante el implante de prótesis de pene. Análisis de nuestra serie. Actas Urol Esp 2004;28(8):575-580.

Dr. G. Atienza Merino

Axencia de Avaliación de Tecnoloxías

Sanitarias de Galicia (avalia-t)

Consellería de Sanidade

Edif. Administrativo de San Lázaro s/n

15781- Santiago de Compostela

E-mail : gerardo.atienza.merino@sergas.es

(Trabajo recibido el 24 de mayo 2005) 